\title{
In-situ catalyst mediated growth and self-doped silicon nanowires for use in nanowire solar cells
}

\author{
Krishna Nama Manjunatha ${ }^{a}$, Shashi Paul ${ }^{\text {a* }}$ \\ ${ }^{a}$ Emerging Technologies Research Center, De Montfort University, The gateway, Leicester,LE18BH,United Kingdom
}

\begin{abstract}
We report the growth of self-doped silicon nanowires (SiNWs), using gallium and bismuth catalysts, by employing a vapour-liquid-solid method. This enables the formation of both $\mathrm{p}$ - and $\mathrm{n}$-doped nanowires without the use of expensive and toxic gases that are conventionally used and opens a new route to a simplified and cost effective process for doping SiNWs. The chosen catalysts have the lowest eutectic temperature available for the growth of silicon nanowires. The growth of self-doped SiNWs, for the fabrication of photovoltaic cells, has been demonstrated for the first time in this study.
\end{abstract}

Keywords: self-doping, eutectic temperature, silicon nanowires, photovoltaic solar cells, light trapping.

Abbreviations: a-Si:H - amorphous silicon, SiNWs - silicon nanowires, PECVD - plasma enhanced chemical vapor deposition, $\mathrm{Ga}$ - gallium, $\mathrm{Bi}$ - bismuth, VLS - vapor-liquid-solid. 


\section{Introduction}

There are ongoing attempts to bring down the cost per watt of photovoltaic solar cells by increasing their efficiency and cutting the fabrication cost. The possible routes to increase in the efficiency are: surface texturing, antireflection coating, use of black silicon, plasmonics [1-4], or fabrication of solar cells using alternative materials that are more inexpensive compared to silicon ( $\mathrm{Si}$ ) solar cells. Albeit, there are a number of upcoming materials to replace Si in solar cell industries, Si-based solar cells have the largest share of the photovoltaic market [5]. The average cost of a Si solar cell module in 2015 was $\$ 0.6 / \mathrm{W}$, compared to $\$ 1.9 / \mathrm{W}$ in 2010 [6]. In recent years, a method has been proposed to improve the efficiency of solar cells by light absorption with the use of nanostructures, this includes; nanowires [7], nano-cones [4], and nano-domes [1]. Nanostructured solar cells are gaining more importance these days due to low-cost fabrication, enhanced antireflection (AR) without the need for additional AR coating on the top of the solar cell, light trapping and both a graded and/or high refractive index [8-11].

Plasma-enhanced chemical vapour deposition (PECVD) is a mature manufacturing technology in industries that manufacture amorphous/micro-crystalline Si solar cells. This technique is generally used for deposition of amorphous silicon (a-Si:H), however, using a metal catalyst and exploiting a vapour-liquid-solid (VLS) growth process, it is possible to obtain crystalline Si [11]. This technique has been used to grow Si nanowires. In brief, the VLS growth process involves Si vapour adsorbed onto and diffused into liquid metal droplet when the supersaturated precipitation of $\mathrm{Si}$ atoms is observed as solid crystalline nanowires [11]. The metal catalyst that is employed in the VLS growth process can deliver some advantages if carefully selected. It is already well established that the growth of metal-assisted Si nanowires intake metal atoms in their lattice structures during the growth process [12]. This can be beneficial if the chosen metal ionisation energy level is close to conduction and/or valence band of Si. Such selection of a metal catalyst will contribute to self-doping. Contrary to this, if the ionisation/impurity energy level of metal used as a catalyst such as Gold (Au) is close to the middle of the band-gap, it acts as an effective recombination centre for charge carriers and significantly degrades carrier mobility and lifetime. This will contribute to a decrease in the efficiency of solar cells. Au is commonly used in the growth of SiNWs by PECVD. Therefore, by selecting metals, other than gold, whose ionisation energy levels contribute in doping as well as facilitating the growth of SiNWs will open a new chapter of self-doping. There are established methods to dope SiNWs that include diffusion processes, dissociation and injection of dopants from gaseous precursors (diborane and phosphine) during the nanowire growth [11]. However, doping from a catalyst has several advantages, such as; elimination of toxic gases, avoiding the complexity of counter-doping caused from the catalyst, reducing costs, and simplifying the optimisation process. 
SiNW solar cells fabricated on glass substrates use different junction architectures including P-N and $\mathrm{P}-\mathrm{i}-\mathrm{N}$ junctions. In these architectures, the core nanowire is crystalline and other layers deposited over the core nanowire are amorphous Si. The Examples reported include; a crystalline Si nanowire core is either P/N-type and the junction is formed by the deposition of N/P-type a-Si:H over a core nanowire, efficiency is further improved by introducing intrinsic a-Si:H between the N/P-type crystalline $\mathrm{Si}$ core and the P/N-type a-Si:H layer, which enhances depletion width [11, 13]. a-Si:H is not only used for the formation of a junction, it is also used for the surface passivation of nanowires to minimise the surface defects and recombination of carriers $[10,11]$. However, use of a-Si:H in SiNW solar cells poses several problems including stability and efficiency. In particular, solar cells that utilise a-Si:H suffer from low mobility and conductivity, light-induced degradation, and low carrier diffusion length, which is a consequence of defects within the material $[14,15]$.

In this work, we show in-situ self-doping of SiNWs during growth from an appropriate metal catalyst; both p-type and n-type doping can be achieved. Amongst several metal catalysts, the catalyst that can dope SiNWs, while keeping the eutectic temperature as low as possible $\left(<300{ }^{\circ} \mathrm{C}\right)$ was selected. Nanowires grown from selected catalysts were examined by the Seebeck effect to confirm the doping type. In a nutshell, we discuss the doping of SiNWs from catalysts and the use of doped Si nanowires in photovoltaic solar cells. The use of a-Si:H layers are done away with in this study, which opens new paths to obtain higher efficiency and more stable solar cells. The use of a-Si:H is a possible drawback in SiNW solar cell architectures [11].

\section{Experimental details}

Single side polished doped Si wafers, both n-type (phosphorous doped, 300 $\mu$ m thickness, 8-30 ohm$\mathrm{cm}$, (100) orientations) and p-type (boron doped, 270 $\mu \mathrm{m}$ thickness, 1-20 ohm-cm, 100 orientations), were used for the fabrication of the solar cells. Si wafers and glass substrates (corning - 2855) were cleaned using an RCA process and the native oxide on Si wafers was removed by immersing wafers in a buffered hydrofluoric (HF) acid solution for 8 seconds, then the residual HF is removed by rinsing thoroughly in deionised water. Aluminium was evaporated onto the unpolished side of the $\mathrm{p}-\mathrm{Si}$ wafer and gold-antimony alloy was evaporated onto the unpolished side of the n-Si wafer at $0.1 \mathrm{~nm} / \mathrm{s}$ at $5 \times 10^{-6}$ mbar base pressure to obtain a 250nm thick layer This was annealed in a vertical furnace at $500{ }^{\circ} \mathrm{C}$ for 30 minutes in a nitrogen atmosphere to establish ohmic contact. Later, Si and glass substrates were loaded into a thermal evaporator to deposit $5 \mathrm{~nm}$ thin films of $\mathrm{Ga}$ and $\mathrm{Bi}$ at $0.1 \mathrm{~nm} / \mathrm{s}$ at $5 \times 10^{-6}$ mbar base pressure. Thickness was measured using a quartz microbalance. Si and glass coated with $\mathrm{Ga}$ and $\mathrm{Bi}$ were loaded into a capacitively-coupled RF-PECVD chamber and subjected to an $\mathrm{H}_{2}$ plasma at $400{ }^{\circ} \mathrm{C}$, with an $\mathrm{H}_{2}$ flow rate of $100 \mathrm{sccm}$ (standard cubic centimetre per minute), 25W RF power and chamber pressure $260 \mathrm{mbar}$ for $180 \mathrm{~s}$ to form catalyst nanoparticles. Immediately after this, $\mathrm{SiH}_{4}$ gas was introduced at $20 \mathrm{sccm}$ with the RF power reduced to $10 \mathrm{~W}$ while maintaining the same pressure for a duration of 15 minutes to grow the SiNWs. After cooling down the PECVD chamber to 
room temperature, the substrates with SiNWs were loaded into the vacuum thermal evaporator to deposit a 500nm thick Ag top electrode under the same conditions as discussed before to complete the fabrication of a solar cell. Finally, fabricated solar cells are rapidly annealed wherein the substrate temperature was maintained at $1000^{\circ} \mathrm{C}$ for 4 seconds using a tungsten filament heater in the vacuum evaporator which was at $10^{-7} \mathrm{mbar}$ base pressure.

A Seebeck experiment was performed to determine the type of doping from the catalyst for the SiNWs. The in-house experimental setup comprised of; two Peltier cells, a heat sink, a pico-ammeter (HP-4140B), thermometers, and a pyrometer (for monitoring the temperature). In this experimental system, one Peltier module is used to generate a heated plate and the other to generate a cooled plate. Within the Seebeck experiment, the generation of the voltage is determined by the difference in the metal responses. Here both electrodes were $500 \mathrm{~nm}$ thick Al electrodes (100 $\mu \mathrm{m}$ gap between electrodes), and so the observed potential difference will be negligible. Due to the fact that very low currents pass in SiNWs, a pico-ammeter with a resolution of $1 \mathrm{fA}$ was used. In this experiment, the temperature of the first $\mathrm{Al}$ electrode (cold side) was maintained at $-5{ }^{\circ} \mathrm{C}$ and the other $\mathrm{Al}$ electrode (heated side) was maintained at $+60{ }^{\circ} \mathrm{C}$. Reflectance measurements were performed using a Thermoscientific UV-Vis-NIR evolution 300 spectrometer equipped with $45^{\circ}$ fixed angle specular reflectance accessory purchased from Pike Technologies. The spectrometer resolution is $0.5 \mathrm{~nm}$ and it is fitted with a xenon light source.

\section{Results and Discussion}

A few studies have shown that tapered nanowires with the absence of the metal catalyst, at the tip of the nanowires, are attributed to the evaporation of the metal during growth, etching of metal by formation of metal-hydrides in the presence of $\mathrm{H}$-radicals, or the incorporation of the metal atoms in the nanowires $[13,16,17]$. Using atom probe tomography, a single nanowire grown from In and Sn was studied experimentally to understand and provide evidence for the incorporation of catalyst atoms in SiNWs growth via a PECVD process [12]. It is advantageous when the incorporated metal atoms in Si can contribute to doping. As shown in Fig.1, a few elements create an ionisation energy level close to the valence or conduction-band edges of Si. Elements such as $\mathrm{Sb}, \mathrm{P}, \mathrm{As}$, and B are generally used for doping Si wafers. Considering only those metals that have the potential to grow SiNWs; metals such as $\mathrm{Bi}, \mathrm{Mg}$, and $\mathrm{Ti}$ can introduce an impurity level close to the conduction band edge of Si that facilitates n-type doping and metals such as $\mathrm{Al}, \mathrm{Ga}$, and In can introduce an impurity level closer to the valence band edge of Si that facilitates p-type doping. To dope SiNWs we have considered Bi as a catalyst to grow n-doped SiNWs and Ga as a catalyst to grow p-doped SiNWs, because of their low eutectic temperature with $\mathrm{Si}$ (for $\mathrm{n} / \mathrm{p}$ type doping), the existence of one ionisation energy level compared with two energy levels introduced by $\mathrm{Mg}$ and because the ionisation energy levels are much closer to the conduction-band edge as compared with other metals (Fig.1). 


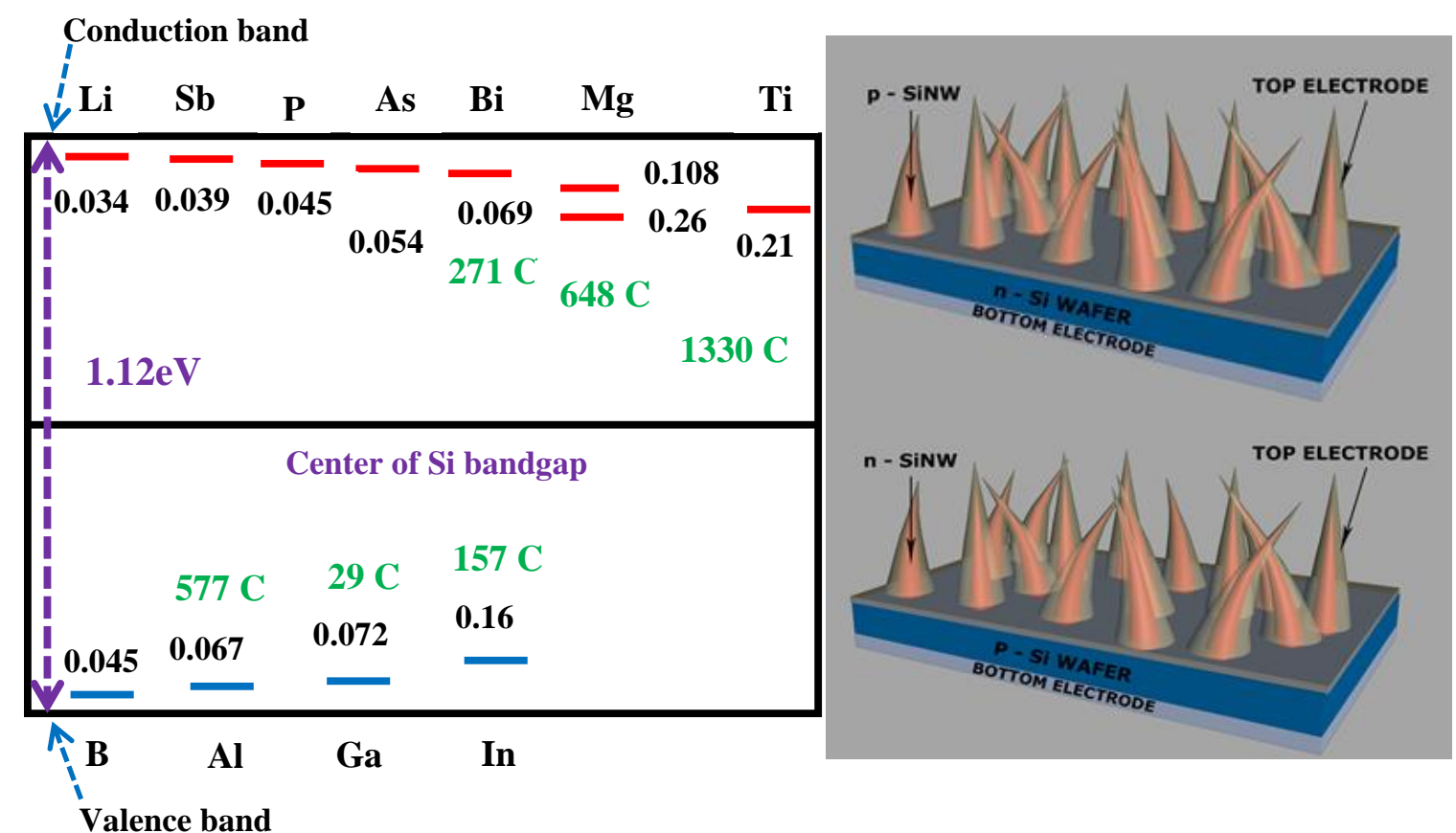

Fig.1. Ionisation energy levels in the forbidden gap of Si introduced by impurities [18], Metals that have a potential to grow SiNWs are indicated with their eutectic temperature (left). Illustration of p-n junction SiNW solar cells from Ga and Bi mediated SiNWs fabricated in this work (right).

The incorporation of a catalyst in SiNWs is more prominent when the growth temperatures are higher than the eutectic temperature of the alloy, and during faster growth rate of nanowires $[12,13]$. Hence, growth temperature in this work was maintained at $400{ }^{\circ} \mathrm{C}$, which is higher than the eutectic temperature of $\mathrm{Bi}$ and $\mathrm{Ga}$ with $\mathrm{Si}$. Recently, $\mathrm{Yu}$ et al experimentally demonstrated simultaneous $\mathrm{Bi}$ assisted growth and doping of SiNWs without the use of dopant gases and showed its potential in the fabrication of radial junction SiNW solar cells [13]. Generally, semiconductor electronic devices require a controlled doping. The doping established in the VLS process can be quantitatively controlled by varying the catalyst size (amount of metal atoms available in a catalyst droplet) that is available to assist growth.

\subsection{Bismuth-catalysed SiNWs}

Bi metal is used as a catalyst to grow $\mathrm{SnS}_{2}$ nanotubes [17], germanium nanowires [16], and selfcatalysed bismuth whiskers and nanowires [19]. Recently, Bi has been exploited for the growth of SiNWs [20]. It is an interesting prospect as a catalyst due to its low eutectic temperature of $271.4{ }^{\circ} \mathrm{C}$ with $\mathrm{Si}$ and it brings out a shallow donor level $(0.069 \mathrm{eV}$ below the conduction-band edge of $\mathrm{Si})$. There are few reports on the growth of SiNWs with Bi as a catalyst. This may be due to several challenges, including; the quick oxidation of bismuth [16], the high vapour pressure $[13,17,20]$, the low Si solubility [21], and the low surface tension [11]. The growth of SiNWs from Bi is better understood with a binary phase diagram that explains the VLS growth process. As seen in Fig.2, only 
one eutectic temperature exists at $271.4{ }^{\circ} \mathrm{C}$, which is the same as the Bi melting point with a concentration of $\mathrm{Si}$ in a liquid alloy at a eutectic point of $<10^{-5}$ at $\%$. However, there exists a steep liquefied line where $\mathrm{Si}$ is supersaturated in a liquid alloy from 271.4 up to $1000{ }^{\circ} \mathrm{C}$. For a growth process of $\mathrm{SiNWs}, \mathrm{SiH}_{\mathrm{x}}$ radicals are adsorbed onto the surface of $\mathrm{Bi}$ droplets and $\mathrm{Si}$ atoms are absorbed into to the liquid Bi droplet and precipitate at the interface between the substrate and the liquid alloy droplet. From the eutectic diagram, it is clear that the growth process can occur at temperatures higher than the eutectic temperature. However, owing to the high vapour pressure of $\mathrm{Bi}$, growth at higher temperatures is challenging due to the evaporation of $\mathrm{Bi}$. The spectral reflectivity (Fig.2) of $\mathrm{Si}$ is compared after the deposition of a catalyst and the growth of SiNWs. Experimentally measured reflectance of a polished silicon wafer is compared with the optically modelled response with the aid of OPAL2 simulation software to verify the experimental setup. The measured reflectance is approximately $3 \%$ higher than the calculated response. The reflectivity increases after depositing the catalyst, which is possible due to the fact that the property of the high reflectivity shown by metals is inherited. Interestingly, the reflectivity decreases to less than $20 \%$ for all wavelengths, from visible to near-infrared, after the growth of SiNWs.
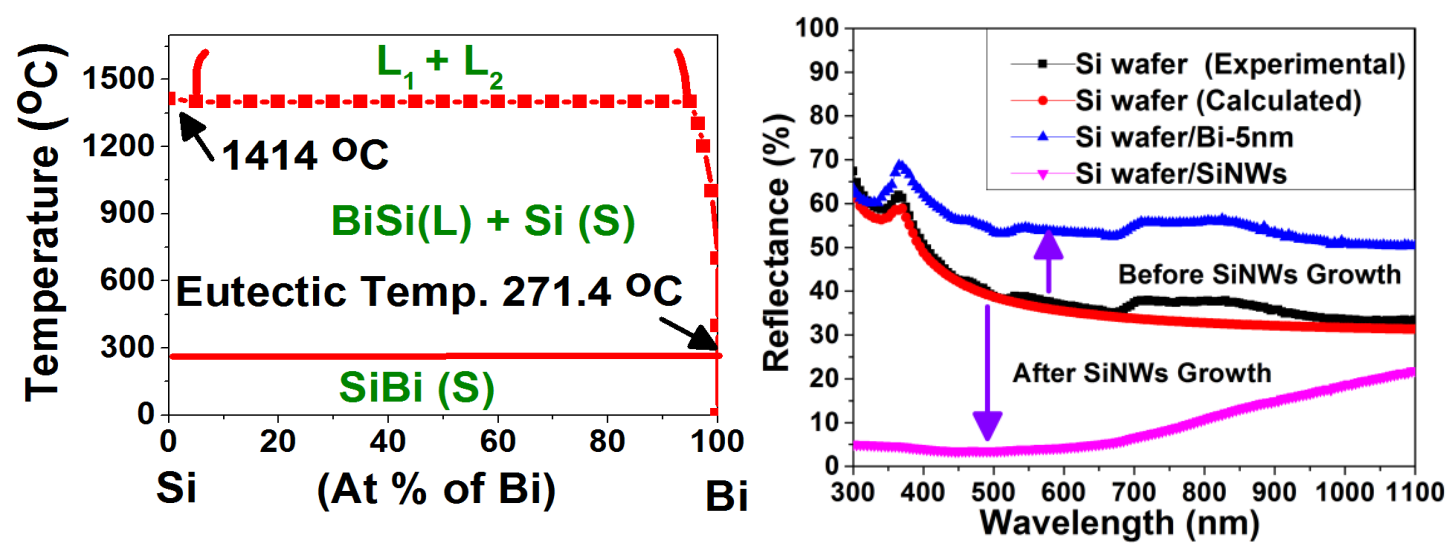

Fig.2. Binary phase diagram of Bi-Si alloy reproduced from [21] (Left). Reflectance of Si wafer depicting increased reflectance in Si coated with Bi catalyst and reduced reflectance due to efficient light absorption in SiNWs (right).

According to Nebol's criterion, it is difficult to obtain a unidirectional growth of SiNWs from low surface tension metals. Later, Schmit, et al showed the minimum surface tension of a liquid metal catalyst to be $0.85 \mathrm{~J} / \mathrm{m}^{2}$ to attain a stable VLS growth of SiNWs. Therefore, SiNW growth from Bi is puzzling due to the low surface tension of about $\sim 0.36 \mathrm{~J} / \mathrm{m}^{2}$. Later, Yu et al [11] described a growth process of nanowires in low surface tension metals considering the Sn catalyst that can be implied to all low surface tension metals (including Bi used in this work). Fig.3 depicts the morphology of SiNWs grown from a Bi catalyst that are better separated and appear to be straight. However, these nanowires are tapered, which can be ascribed to the reduction in the size of the catalyst during growth that reduces the region of the $\mathrm{Si}$ precipitation. Shrinkage of the catalyst is observed owing to; 1 ) formation of volatile metal hydrides by reaction with H-radicals from plasma, 2) the high rate of 
evaporation of $\mathrm{Bi}$ due to the high vapour pressure at temperatures exceeding $350{ }^{\circ} \mathrm{C}$, which is assisted by the abnormal Arrhenius behaviour of Bi from $350{ }^{\circ} \mathrm{C}$ to 1000 [19], and 3) wetting of sidewall of SiNWs, a general problem posed by SiNWs grown from low surface tension metal catalyst [11] and diffusion of Bi in SiNWs.
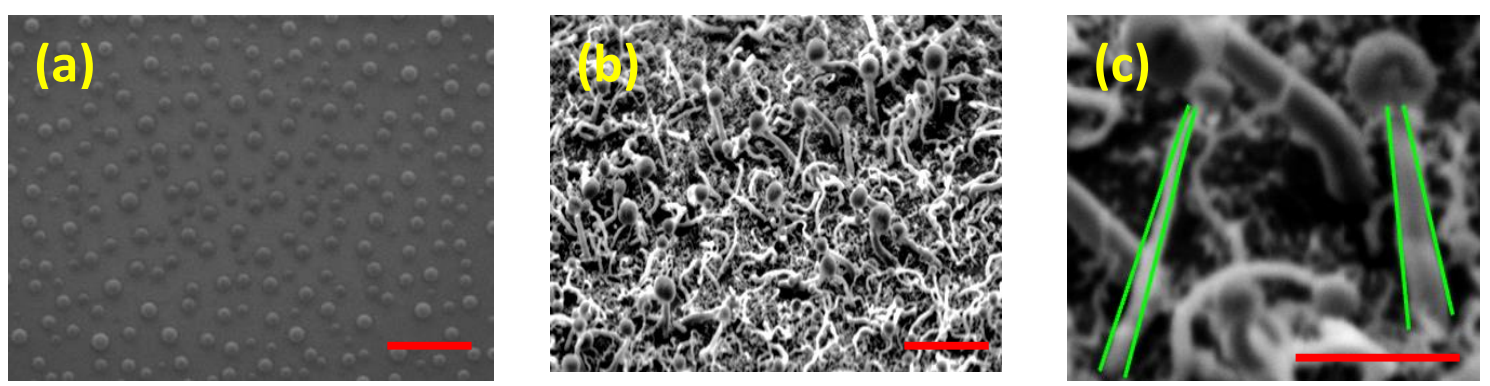

Fig. 3. Formation of Bi nanoparticles after $\mathrm{H}_{2}$-plasma treatment (a), SiNWs grown from Bi following VLS process with presence of catalyst on top (b), high magnification image showing tapered SiNWs (c). All scale bars in SEM images are $1 \mu \mathrm{m}$.

The vapor pressure of an element $(p)$ determines the rate of evaporation as a function of pressure at the desired temperature [16]:

$$
\Delta p=\frac{2 \Upsilon M}{r \rho R T} p_{0}(T)
$$

Where $Y$ is the surface tension of a metal particle, $T$ is the temperature, $M$ is the atomic mass of a metal, $\mathrm{r}$ is the radius of the particle, $\rho$ is the density, $\mathrm{R}$ is the Arrhenius constant and $P_{o}(T)$ is the vapour pressure of the metal particle. As shown in equation (1), the surface tension is directly proportional to the vapour pressure. Considering the low surface tension of bismuth-silicon alloy system, initially, it would not tend to vaporise at faster rates at a given temperature and pressure. However, after $\mathrm{H}_{2}$-plasma treatment, the nanoparticles of $\mathrm{Bi}$ would gain surface tension due to changes in their morphology to spheres (to minimise surface energy), where vaporisation of $\mathrm{Bi}$ increases. As nanowires grow, $\mathrm{Bi}$ is constantly dissolved into SiNWs while etched by $\mathrm{H}$-radicals that cause the reduction in their size. At this stage, the vaporisation of $\mathrm{Bi}$ is drastically increased due to the smaller radius of Bi particles, which can be deduced from the equation (1), where the radius of the particle is inversely proportional to the vapour pressure. This clearly indicates that the growth process needs to be optimised with proper consideration for all stages of the PECVD growth process.

\section{$\underline{3.2 \text { Gallium Catalyzed SiNWs }}$}

Ga is extensively studied as a catalyst to grow nanostructures, including SiNWs, for applications in; charge storage, solar cells, and gas sensors [22, 23]. It has a single eutectic temperature, as low as $29.7^{\circ} \mathrm{C}$, with a steep liquefied line where it is possible to supersaturate $\mathrm{Si}$ in a liquid alloy from the eutectic temperature $\left(29.7^{\circ} \mathrm{C}\right.$ ) up to $500{ }^{\circ} \mathrm{C}$ (Fig. 4). The ionisation energy of gallium in the forbidden gap of $\mathrm{Si}$ is about $0.072 \mathrm{eV}$ (donor level) above the valence band that acts as a p-type 
impurity in $\mathrm{Si}$. The growth process is similar to that explained earlier for the Bi catalyst, which relies on the VLS growth process. As with $\mathrm{Bi}$, the Si concentration in $\mathrm{Ga}$ is very low about $<10^{-8}$ at\%. In contrast, the Ga catalyst does not suffer from the problems posed by the Bi catalyst, the vapour pressure of gallium is low and the surface tension $\left(\sim 0.71 \mathrm{~J} \mathrm{~m}^{-2}\right)$ is higher compared with all low surface tension metals (In, Sn and Bi) that are used for SiNW growth. Spectral reflectivity in Fig.4 depicts reduced reflection from Si substrates with SiNWs which could lead to better absorption of light.
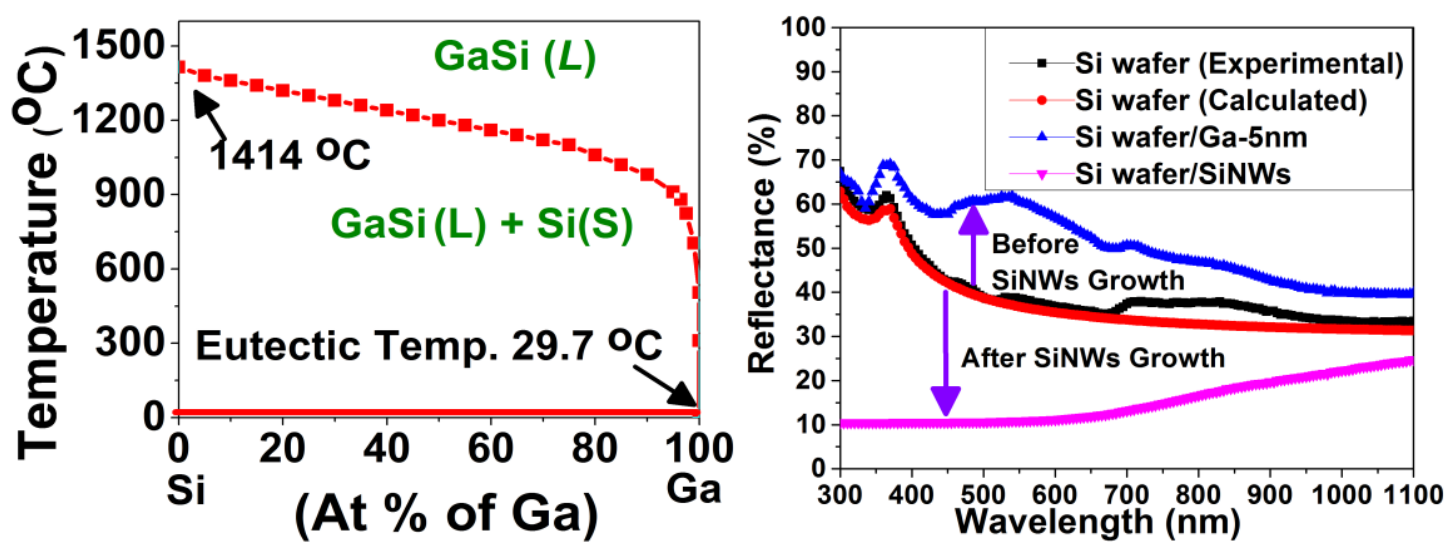

Fig.4. Binary phase diagram of Ga-Si alloy reproduced from [24](Left). Reflectance of Si wafer depicting increased reflectance in Si coated with Ga catalyst and reduced reflectance after SiNW growth due to efficient light absorption in SiNWs (right).

Ga mediated SiNW growth shows better morphology with dense and vertical nanowires (Fig.5). This can be clearly understood by the surface energy difference for Ga-Si alloy, which is $0.1 \mathrm{~J} / \mathrm{m}^{2}$ to obtain a stable and vertical growth of nanowires as predicted by Schmit et al. The average length of nanowires is $3 \mu \mathrm{m}$ and the density of nanowires calculated from the image-J software is $6.3 \times 10^{7} \mathrm{~cm}^{-}$ 2.
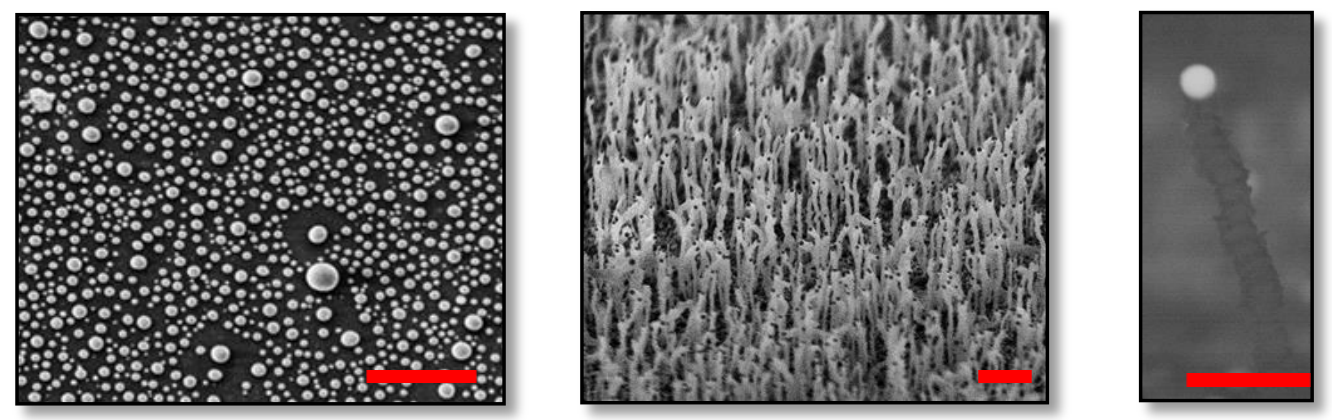

Fig. 5. Formation of Ga nanoparticles after $\mathrm{H}_{2}$-plasma treatment (a),45 tilted image of SiNWs grown from $\mathrm{Ga}$ following VLS process with the presence of catalyst on top (b), backscattered electron image of individual Ga mediated SiNW with a catalyst on top (c). All scale bars in SEM images are $1 \mu \mathrm{m}$. 


\section{Characterisation of SiNWs and Solar cells}

Seebeck experiments were performed to determine properties such as the dopant type in semiconductors and to gather properties of thermoelectric devices [25-28]. This kind of experiment is performed for large scale devices not excluding nanostructures to understand the thermoelectric properties in $\mathrm{ZnO}$ nanowires [26], SiNWs [27, 28], and Si-nanoparticles [25]. The experimental setup used in this work is similar. However, a detailed investigation was not performed, as the research focus is not to understand the thermoelectric properties of SiNWs. Instead, it focuses on determining if the catalyst contributed to the doping of SiNWs and to understand the dopant type $(\mathrm{n} / \mathrm{p})$. By convention, semiconductors are determined to be p-type when holes diffuse from the cooled to the heated side of the device. Moreover, when electrons diffuse from the hotter to the cooler side, they are determined as n-type. Hence, one can easily determine the semiconductor type by measuring the current or the voltage across the semiconductor which is subjected to a temperature gradient. In this work, the current is measured to determine the type of semiconductor, in contrast, the semiconductor is n-type when a positive value of current is measured and p-type when a negative value of current is measured. To measure the current flowing in SiNWs due to temperature gradients, SiNWs grown on glass substrates were covered by Al gap electrodes. A current could be measured, but not for all the samples with SiNWs. This is attributed to the growth morphology of the nanowires not being the same for every sample, conformal metal coating on the top of the nanowires is challenging for uneven nanowires and if the grown nanowires are perpendicular, unless they touch each other there would not be a path for the current flow. Hence, the glass substrates that are initially coated with Al gap electrodes are used and scratched SiNWs are sprinkled at the gap between the electrodes for analysis. The currents measured for SiNWs grown from $\mathrm{Bi}$ and $\mathrm{Ga}$ catalyst were $+430 \mathrm{pA}$ and $-154 \mathrm{pA}$ respectively. This clearly indicates that nanowires are doped from catalyst atoms and confirms that Bi-mediated SiNWs are n-type while Ga-mediated SiNWs are p-type.
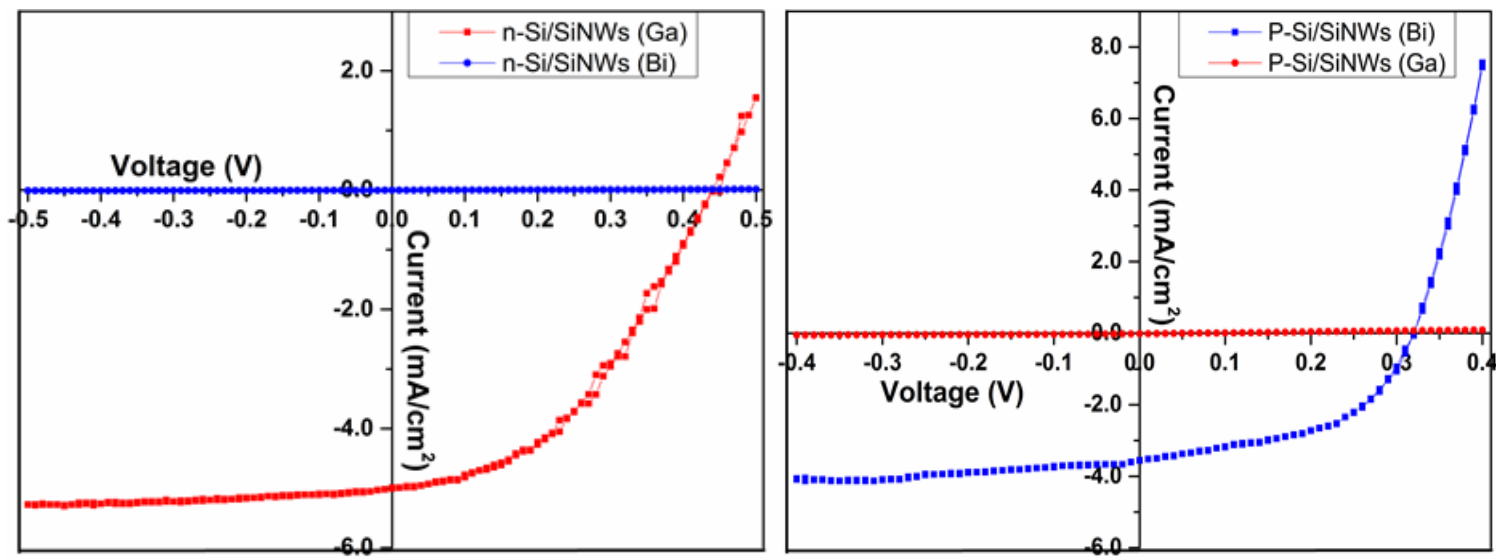

Fig. 6. I-V characteristics of corresponding SiNW solar cells on both $n$-Si (left) and p-Si (right) substrates. 
$\mathrm{Bi}$ and Ga catalysed SiNWs are grown on p- and n-type Si wafers to further understand doping from the catalyst atoms and to fabricate solar cells. Considering both Ga and Bi mediated SiNWs grown on $\mathrm{p}$ and $\mathrm{n}-\mathrm{Si}$ wafers, the junction is established better for Bi catalysed SiNWs on p-Si substrate and Ga catalysed SiNWs on an n-type Si substrate. With observation of rectifying behaviour and solar cell characteristics depicting $\mathrm{V}_{\text {oc }}$ (open circuit voltage) and $\mathrm{I}_{\mathrm{sc}}$ (short circuit current) in I-V curves (Fig.6) it is clear that only diffusion of catalyst atoms into SiNWs during growth is contributing to doping nanowires. Doped nanowires have established a junction signifying that SiNWs obtained from Bi and Ga are n-doped and p-doped, respectively. With this observation and Seebeck experimental conclusions, the authors believe that diffused catalyst atoms take part in doping nanowires. A few studies have shown injection of catalyst atoms in nanowire growth by studying single nanowire grown from In, Sn and Al using atom-probe tomography $[12,29]$. Recently, Yu et al demonstrated radial junction SiNW solar cells with Bi-mediated SiNWs establishing a junction with boron-doped a-Si:H demonstrating nanowires are $n$-doped from the catalyst [13]. Along with experimental conclusions from atom-probe tomography, radial junction solar cells, and our experimental results, it suggests that diffused catalyst atoms contribute to the doping of nanowires.

\begin{tabular}{|l|l|l|l|l|}
\hline & $\begin{array}{l}\mathbf{V}_{\text {oc }} \\
(\mathbf{V})\end{array}$ & $\begin{array}{l}\mathbf{I}_{\mathbf{s c}} \\
\left(\mathbf{m A} / \mathbf{c m}^{2}\right)\end{array}$ & $\begin{array}{l}\mathbf{F F} \\
(\%)\end{array}$ & $\begin{array}{l}\mathbf{E f f} \\
(\boldsymbol{\%})\end{array}$ \\
\hline p-Si-SiNWs(Bi) & 0.32 & 3.64 & 50.1 & 5.8 \\
\hline p-Si-SiNWs(Ga) & 0.065 & 0.015 & - & - \\
\hline n-Si-SiNWs(Ga) & 0.43 & 5.12 & 43.2 & 9.2 \\
\hline n-Si-SiNWs(Ga) & 0.040 & 0.002 & - & - \\
\hline
\end{tabular}

Fig. 7. Extracted parameters of SiNW solar cells tested under Oriel-150W light source whose output is $10 \mathrm{~mW} / \mathrm{cm}^{2}$ (left). Top view of solar cells with SiNWs covered with top contact (right).

Measured $I_{s c}$ and $V_{o c}$ are comparable to traditionally doped SiNW solar cells with an efficiency of $5.8 \%$ and $9.2 \%$, for Bi and Ga-doped SiNWs respectively (Fig. 7). The measured efficiency is higher in Ga-based SiNW solar cells due to the dense growth of nanowires, that enhances light trapping and absorption. Better performance of solar cells fabricated from SiNWs grown from Ga compared to Bi is also attributed to the higher $\mathrm{Si}$ volume fraction obtained from dense growth that contributes to a higher number of photo-generated carriers. Light absorption does not heavily depend on the arrangement (periodical, hexagonal, or square) of nanowire arrays, but it increases logarithmically with increasing length in the nanowires [30]. SiNWs grown from $\mathrm{Ga}$ and $\mathrm{Bi}$ are approximately $3 \mu \mathrm{m}$ and $1 \mu \mathrm{m}$ long, respectively, which is another key parameter that has shown higher efficiency for solar cells with SiNWs grown from Ga. However, the choice of catalyst is important if doping has to be achieved; not all catalysts take part in doping. This is understood with the impurity level in the forbidden gap of $\mathrm{Si}$. Some metals ( $\mathrm{Au}, \mathrm{Cu}$ and $\mathrm{Zn}$ ) have ionisation energy levels close to the middle of Si band-gap [11] that would pose a serious threat in the increasing recombination rate of carriers. 
Hence, these metals are generally excluded for growth of SiNWs used in electronic devices. Conversely, metal catalysts used in this work are beneficial due to the fact that in-situ doping and growth of nanowires at very low temperatures is conceivable. This work also demonstrates the realisation of solar cells utilising crystalline $\mathrm{Si}$ while eliminating a-Si:H layers that are predominantly used in radial junction SiNW solar cells [11,13]. Solar cells fabricated in this work using only C-Si show promise towards the next generation of solar cell, which exploit the quantum properties of nanowires in achieving higher efficiencies and better stability. Studies have demonstrated that colossal injection of catalyst atoms is about four orders of magnitude greater than the equilibrium solubility [29] and the segregation of the diffused catalyst atoms occurs at stacking faults (twin planes, structural defects) in SiNWs [12]. Hence, the optimised doping process from catalyst atoms for uniform distribution in nanowires and controlling their concentration demands more investigation to achieve higher efficiency in solar cells.

\section{Conclusion}

This work has demonstrated the features of a multi-purpose catalyst that not only mediates but controls the growth morphology. Additionally, injection/diffusion of catalyst atoms takes part in doping nanowires. With a series of experiments, including Seebeck and solar cell analysis, it is clear that doping is established from specific metal catalysts whose ionisation energy levels lie in a close proximity to the conduction or valence band. Ga- and Bi-catalysed SiNWs in fabricated solar cells show higher efficiencies of $9.2 \%$ and $5.8 \%$, respectively. This study completely excludes the use of expensive and toxic gases such as phosphine, trimethylborane, and diborane that are conventionally used for doping SiNWs. The catalyst doping simplifies the fabrication process and reduces the final cost. Along with the fabrication of SiNW solar cells, we are able to establish junctions from crystalline Si components excluding a-Si:H layers that are traditionally used in radial junction SiNW solar cells, which pose problems such as higher recombination rate and, importantly, poor stability.

\section{References}

[1] J. Zhu, C. -. Hsu, Z. Yu, S. Fan and Y. Cui, "Nanodome solar cells with efficient light management and self-cleaning," Nano Lett., vol. 10, pp. 1979-1984, 2010.

[2] S. R. Marthi, S. Sekhri and N. M. Ravindra, "Optical Properties of Black Silicon: An Analysis," JOM, vol. 67, pp. 2154-2159, 2015.

[3] Y. Zhang, N. Stokes, B. Jia, S. Fan and M. Gu, "Towards ultra-thin plasmonic silicon wafer solar cells with minimized efficiency loss," Sci. Rep., vol. 4, 2014.

[4] S. Huang, J. W. M. Lim, C. S. Chan, S. Xu, D. Wei, Y. Guo, L. Xu and K. Ostrikov, "Scalable Production of Silicon Nanocone Solar Cells in Integrated Plasma Photovoltaic Nanofabrication Cluster," Plasma Processes Polym., vol. 13, pp. 161-169, 2016. 
[5] T. Saga, "Advances in crystalline silicon solar cell technology for industrial mass production," NPG Asia Mater., vol. 2, pp. 96-102, 2010.

[6] A. Louwen, W. Van Sark, R. Schropp and A. Faaij, "A cost roadmap for silicon heterojunction solar cells," Sol Energ Mater Sol Cells, vol. 147, pp. 295-314, 2016.

[7] M. Jeon and K. Kamisako, "Synthesis and characterization of silicon nanowires using tin catalyst for solar cells application," Mater Lett, vol. 63, pp. 777-779, 2009.

[8] M. Hasan, M. Huq and Z. Mahmood, "A review on electronic and optical properties of silicon nanowire and its different growth techniques," SpringerPlus, vol. 2, pp. 1-9, 04/10, 2013.

[9] Y. -. Hung and S. -. Lee, "Manipulating the antireflective properties of vertically-aligned silicon nanowires," Sol Energ Mater Sol Cells, vol. 130, pp. 573-581, 2014.

[10] K. Peng and S. Lee, "Silicon Nanowires for Photovoltaic Solar Energy Conversion," Adv Mater, vol. 23, pp. 198-215, 2011.

[11] S. Misra, L. Yu, W. Chen, M. Foldyna and P. Roca I Cabarrocas, "A review on plasma-assisted VLS synthesis of silicon nanowires and radial junction solar cells," J. Phys. D, vol. 47, 2014.

[12] W. Chen, L. Yu, S. Misra, Z. Fan, P. Pareige, G. Patriarche, S. Bouchoule and P. R. I. Cabarrocas, "Incorporation and redistribution of impurities into silicon nanowires during metalparticle-assisted growth," Nature Communications, vol. 5, 2014.

[13] L. Yu, F. Fortuna, B. O'Donnell, T. Jeon, M. Foldyna, G. Picardi and P. Roca I Cabarrocas, "Bismuth-catalyzed and doped silicon nanowires for one-pump-down fabrication of radial junction solar cells," Nano Letters, vol. 12, pp. 4153-4158, 2012.

[14] F. Meillaud, M. Boccard, G. Bugnon, M. Despeisse, S. Hänni, F. -. Haug, J. Persoz, J. -. Schüttauf, M. Stuckelberger and C. Ballif, "Recent advances and remaining challenges in thin-film silicon photovoltaic technology," Materials Today, vol. 18, pp. 378-384, 9, 2015.

[15] M. Fehr, A. Schnegg, B. Rech, O. Astakhov, F. Finger, R. Bittl, C. Teutloff and K. Lips, "Metastable defect formation at microvoids identified as a source of light-induced degradation in a Si: H," Phys. Rev. Lett., vol. 112, 2014.

[16] Y. Xiang, L. Cao, J. Arbiol, M. L. Brongersma and A. Fontcuberta I Morral, "Synthesis parameter space of bismuth catalyzed germanium nanowires," Appl. Phys. Lett., vol. 94, 2009.

[17] A. Yella, E. Mugnaioli, M. Panthöfer, H. A. Therese, U. Kolb and W. Tremel, "Bismuthcatalyzed growth of SnS2 nanotubes and their stability," Angew. Chem. Int. Ed., vol. 48, pp. 64266430, 2009.

[18] S. M. Sze, Physics of Semiconductor Devices. 1981.

[19] S. A. Stanley, C. Stuttle, A. J. Caruana, M. D. Cropper and A. S. O. Walton, "An investigation of the growth of bismuth whiskers and nanowires during physical vapour deposition," J. Phys. D, vol. $45,2012$.

[20] R. R. Kumar, K. N. Rao and A. R. Phani, "Bismuth catalyzed growth of silicon nanowires by electron beam evaporation," Mater Lett, vol. 82, pp. 163-166, 2012. 
[21] Olesinski, R. W., Abbaschian,G.J., "The Bi-Si (Bismuth-Silicon) system," Bulletin of Alloy Phase Diagrams Bulletin of Alloy Phase Diagrams, vol. 6, pp. 359-361, 1985.

[22] N. Gabrielyan, K. Saranti, K. N. Manjunatha and S. Paul, "Growth of low temperature silicon nano-structures for electronic and electrical energy generation applications," Nanoscale Research Letters, vol. 8, pp. 1-7, 2013.

[23] B. S. Simpkins, L. M. Ericson, R. M. Stroud, K. A. Pettigrew and P. E. Pehrsson, "Gallium-based catalysts for growth of GaN nanowires," J. Cryst. Growth, vol. 290, pp. 115-120, 4/15, 2006.

[24] Olesinski, R. W., Kanani, N., Abbaschian,G.J., "The Ga-Si (Gallium-Silicon) system," Bulletin of Alloy Phase Diagrams Bulletin of Alloy Phase Diagrams, vol. 6, pp. 362-364, 1985.

[25] S. P. Ashby, J. García-Cañadas, G. Min and Y. Chao, "Measurement of thermoelectric properties of phenylacetylene-capped silicon nanoparticles and their potential in fabrication of thermoelectric materials," J Electron Mater, vol. 42, pp. 1495-1498, 2013.

[26] G. Kaltsas, C. Tsamis, S. Dalola, G. Faglia, E. Comini, M. Ferroni, C. Soldano, D. Zappa, V. Ferrari and G. Sberveglieri. Seebeck effect in $\mathrm{ZnO}$ nanowires for micropower generation. Procedia Engineering 25pp. 1481-1484. 2011.

[27] G. Schierning, "Silicon nanostructures for thermoelectric devices: A review of the current state of the art," Phys. Status Solidi A Appl. Mater. Sci., vol. 211, pp. 1235-1249, 2014.

[28] G. Pennelli, "Review of nanostructured devices for thermoelectric applications," Beilstein J. Nanotechnology, vol. 5, pp. 1268-1284, 2014.

[29] O. Moutanabbir, D. Isheim, H. Blumtritt, S. Senz, E. Pippel and D. N. Seidman, "Colossal injection of catalyst atoms into silicon nanowires," Nature, vol. 496, pp. 78-82, 2013.

[30] M. Foldyna, L. Yu, S. Misra and P. Roca I Cabarrocas, "Light trapping enhancement in ordered and disordered silicon nanowire based solar cells," in Proceedings of SPIE - the International Society for Optical Engineering, 2013, . 